\title{
Efficacy of a 4-Week Swallowing Rehabilitation Program Combined With Pyriform Sinus Ballooning in Patients With Post-stroke Dysphagia
}

\author{
Yong Kyun Kim, MD, PhD, Kyun Yeon Lee, MD, Sang-Heon Lee, MD
}

Department of Physical Medicine and Rehabilitation, Myongji Hospital, Seonam University College of Medicine, Goyang, Korea

Objective To assess the efficacy of a 4-week swallowing rehabilitation program combined with pyriform sinus ballooning in patients with post-stroke dysphagia (PSD).

Methods We evaluated a total of 30 patients $(n=30)$ with PSD who were admitted to our medical institution between May of 2014 and October of 2016. These patients were randomly assigned to either the trial group ( $\mathrm{n}=15$; a 20-minute conventional rehabilitation followed by a 10-minute pyriform sinus ballooning) or the control group ( $\mathrm{n}=15$; a 30-minute conventional rehabilitation). In these patients, the efficacy outcome measures were pharyngeal remnant, the pharyngeal transit time, the Penetration Aspiration Scale (PAS) scores and the Videofluoroscopic Dysphagia Scale (VDS) scores. We compared the differences in efficacy outcome measures at 4 weeks from baseline between the two groups.

Results There were significant changes in the pharyngeal remnant, the pharyngeal transit time, the PAS scores and the VDS scores at 4 weeks from baseline between the two groups ( $\mathrm{p}<0.0001, \mathrm{p}=0.0001, \mathrm{p}<0.0001$, and $\mathrm{p}=0.0048$, respectively). There were no treatment-emergent adverse events in our series.

Conclusion Our 4-week rehabilitation program combined with pyriform sinus ballooning is an effective and safe modality in patients with PSD. However, further large-scale, long-term, and multi-center studies are needed to corroborate our results.

Keywords Stroke, Deglutition disorders, Rehabilitation, Pharynx, Larynx

Received August 18, 2017; Accepted September 18, 2017

Corresponding author: Sang-Heon Lee

Department of Physical Medicine and Rehabilitation, Myongji Hospital, Seonam University College of Medicine, 55 Hwasu-ro 14beon-gil, Deokyanggu, Goyang 10475, Korea. Tel: +82-31-810-6450, Fax: +82-31-810-5259, E-mail: shf3226@naver.com

ORCID: Yong Kyun Kim (https://orcid.org/0000-0002-1224-8965); Kyun Yeon Lee (https://orcid.org/0000-0001-8822-4006); Sang-Heon Lee (https:// orcid.org/0000-0003-2770-8562).

(c) This is an open-access article distributed under the terms of the Creative Commons Attribution Non-Commercial License (http://creativecommons.org/ licenses/by-nc/4.0) which permits unrestricted noncommercial use, distribution, and reproduction in any medium, provided the original work is properly cited. Copyright $\odot 2018$ by Korean Academy of Rehabilitation Medicine 


\section{INTRODUCTION}

Stroke has been considered the leading cause of death, and its prevalence is higher than that of cancer or cardiovascular diseases [1]. Post-stroke dysphagia (PSD) occurs due to impaired neural control of the oral cavity, pharynx, larynx, and esophagus with an incidence ranging from $19 \%$ to $81 \%[2,3]$. Patients with PSD may exhibit various symptoms such as leakage of food toward the oral cavity, reduced contraction of the pharyngeal muscles, the accumulation of residue in the valleculae and the pyriform sinus, impaired mobility of the hyoid bone and the larynx, abnormal upper esophageal sphincter (UES) opening and a prolonged pharyngeal transit time [4]. They are, therefore, at increased risk of pulmonary complications and mortality $[5,6]$ underscoring the need for a recovery in swallowing function. Growing evidence has shown that prompt detection of PSD is essential to decrease pulmonary complications, shorten length of hospital stay and reduce medical expenses [7].

Treatment modalities for patients with PSD may include compensatory strategies and direct or indirect therapies. Compensatory strategies are used to relieve symptoms without improving swallowing dysfunctions; these include modification of food consistency and postural maneuver (e.g., chin tuck). Direct therapies are associated with diet adjustment. Indirect therapies include stimulation of swallowing or behavioral swallowing interventions. On the other hand, only a limited number of patients with PSD accompanied by cognitive impairment are indicated for conventional exercise interventions such as the Shaker exercise or Mendelsohn maneuver. Furthermore, patients with PSD receiving nasogastric or gastrostomy tube feeding are contraindicated for direct therapies because they are at risk of developing aspiration pneumonia; they are also vulnerable to decreased swallowing frequency as well as dependence on tube feeding [8-13]. It is therefore imperative that novel approaches are developed for the efficient treatment of patients with PSD.

In patients with PSD, pyriform sinus and vallecular space are two sites of residue accumulation [14]. Previous attempts have been made to place a 16-Fr Foley balloon catheter followed by balloon inflation, resulting in recovery of swallowing function. Kim et al. [15] successfully reduced the pharyngeal remnant and pharyngeal transit time by promoting passage of the bolus via relaxation of cricopharyngeal muscles with pyriform sinus ballooning in the videofluoroscopic swallowing study (VFSS). Furthermore, these authors also showed improvements in the post-swallow residue as well as epiglottic mobility after placing a Foley balloon catheter in the vallecular space and then stretching and spreading the epiglottis [16].

In previous studies, however, treatment regimens using a Foley balloon catheter were solely performed during fluoroscopy, which suggests challenges in clinical application. Furthermore, they revealed limitations in continuity and convenience of the treatment. Based on previous studies, we considered using a blind technique without fluoroscopy and placed a Foley balloon catheter till the pharynx. This procedure led to speculation that a ballooning in the pyriform sinus is more advantageous than in the vallecular space. Both sites share similar approach. However, access to the vallecular space was suggested through a U-turn of an endoscope in the oropharynx during transnasal endoscopy. On the other hand, the pyriform sinus can be naturally accessed along the posterior wall of the oropharynx [17].

It is known that a repetitive, task-oriented training is effective for post-stroke rehabilitation [18]. To date, however, the effectiveness of pyriform sinus ballooning based on a blind approach and its long-term effects on the swallowing function are unknown.

The purpose of this study is to evaluate the efficacy of a 4-week course of a conventional dysphagia rehabilitation program combined with a non-fluorosopy-guided pyriform sinus ballooning in patients with PSD.

\section{MATERIALS AND METHODS}

\section{Study patients and setting}

The current single-center, prospective, randomized, observer-blind, controlled study was conducted in a total of 45 patients with PSD who were admitted to our medical institution between May of 2014 and October of 2016 .

Inclusion criteria were as follows: (1) PSD with an onset period of $<3$ years; (2) aspiration or post-swallow residue $(\geq 10 \%)$ in the pyriform sinus at baseline VFSS; and (3) tube feeding at the time of study entry. Exclusion criteria were as follows: (1) acute stroke with an onset period of $<1$ month; (2) swallowing rehabilitation treatment using 
balloon catheterization or botulinum toxin injections within 6 months before study entry; (3) facial deformity or fracture or cervical hyperflexion/hyperextension; (4) vocal cord palsy; (5) airway obstruction or anatomical abnormalities of the pharynx or larynx confirmed on fiberoptic laryngoscope; (6) a history of underlying neurological diseases that may affect swallowing reflex (e.g., Parkinson disease, dementia, or motor neuron disease); and (7) patients considered ineligible for study participation.

After excluding 15 patients, we enrolled a total of 30 in the current analysis. The study was approved by the Institutional Review Board of Myongji Hospital (No. 14-019). All the patients submitted a written informed consent for study participation. The current study was conducted in accordance with the Declaration of Helsinki.

\section{Efficacy outcome measures and assessment}

As previously described, efficacy outcome measures include the following $[12,15,19,20]$.

Pharyngeal transit time (ms): The period of time that a bolus remains in the pharynx, during which food passes from the pharyngeal fornix into the UES.

Pharyngeal remnant (\%): The amount of food retained in the pharyngeal space, whose width corresponds to the area calculated based on the reference value.

Penetration Aspiration Scale (PAS): An 8-point multidimensional indicator of airway invasion, which was used to record the presence of airway penetration (entry of contrast material into the laryngeal vestibule) and aspiration (below the level of the true vocal folds). Videofluoroscopic Dysphagia Scale (VDS): A reliable, objective and quantifiable 100-poins predictor of a longterm, persistent presence of the PSD.

In the current study, changes in the pharyngeal transit time, pharyngeal remnant, PAS and VDS scores at 4 weeks from baseline served as efficacy outcome measures. For efficacy assessment, we compared differences in changes in efficacy outcome measures at 4 weeks from the baseline between the two groups.

\section{Safety assessment}

For safety assessment, we examined whether patients presented with the treatment-emergent adverse events (TEAEs) at the sites of pyriform sinus ballooning.
Patient evaluation and criteria

Depending on whether our patients additionally received pyriform sinus ballooning, they were divided into two groups: the trial group and the control group. Thus, the patients were randomized into either of the two groups using a permuted block design. Swallowing rehabilitation was performed for 30 minutes daily 5 times a week for 4 weeks. In the trial group, a 20-minute conventional treatment was combined with a 10 -minute pyriform sinus ballooning. In the control group, a 30-minute conventional treatment was solely performed.

At baseline, we examined whether the patients met the inclusion/exclusion criteria and analyzed their baseline characteristics-e.g., age, sex, modified Rankin Scale (mRS) scores, National Institutes of Health Stroke Scale (NIHSS) scores, time from the onset of stroke to the VFSS, Korean version of modified Mini-Mental State Examination (K-MMSE) scores, type of feeding, type of stroke and diagnoses. Further, we performed a transnasal endoscopic evaluation of the surface or mucosa using the MAJ922 (Olympus Medical Systems Corp., Tokyo, Japan), to examine the vocal cord movement, laryngopharyngeal reflux, laryngeal sensation and airway patency. In the trial group, we also measured the balloon diameter. We performed a swallowing rehabilitation followed by posttreatment assessment and comparative evaluation of differences in efficacy outcome measures between the two groups (Fig. 1).

\section{VFSS protocol}

The VFSS was performed with a 4-mL bolus of semisolid paste in the chin-tucked position. The semisolid paste was pudding-like without lumps, chunks, or pulp, prepared as a 2:1 volumetric mixture of dysphagia formula level 1 and contrast barium solution (Solotop-HD provided by Taejoon Pharm Company, Yongin, Korea). In addition, $375 \mathrm{~g}$ barium sulfate powder was mixed with 90 $\mathrm{mL}$ water for the contrast solution. In the current study, we used a semisolid whose remnant amount was calculated. Patients with cognitive decline or who had difficulty maintaining head control were seated on a wheelchair, and an assistant held their head to maintain the aforementioned position [12]. All the procedures were recorded on a digital video file and analyzed.

We performed a non-fluoroscopy-guided pyriform sinus ballooning using the VFSS [12]. A 12-Fr Foley balloon 


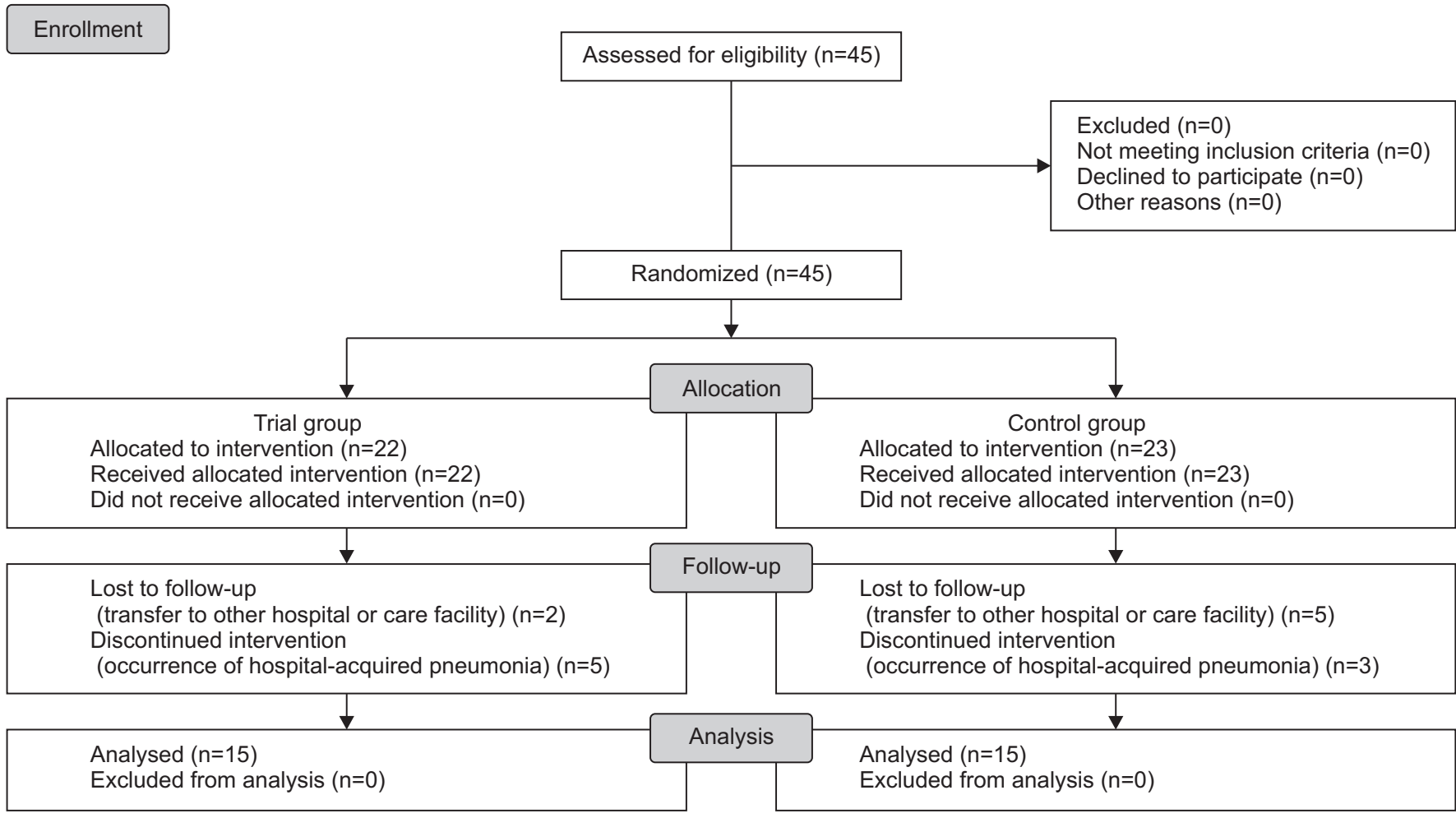

Fig. 1. Study flow chart.
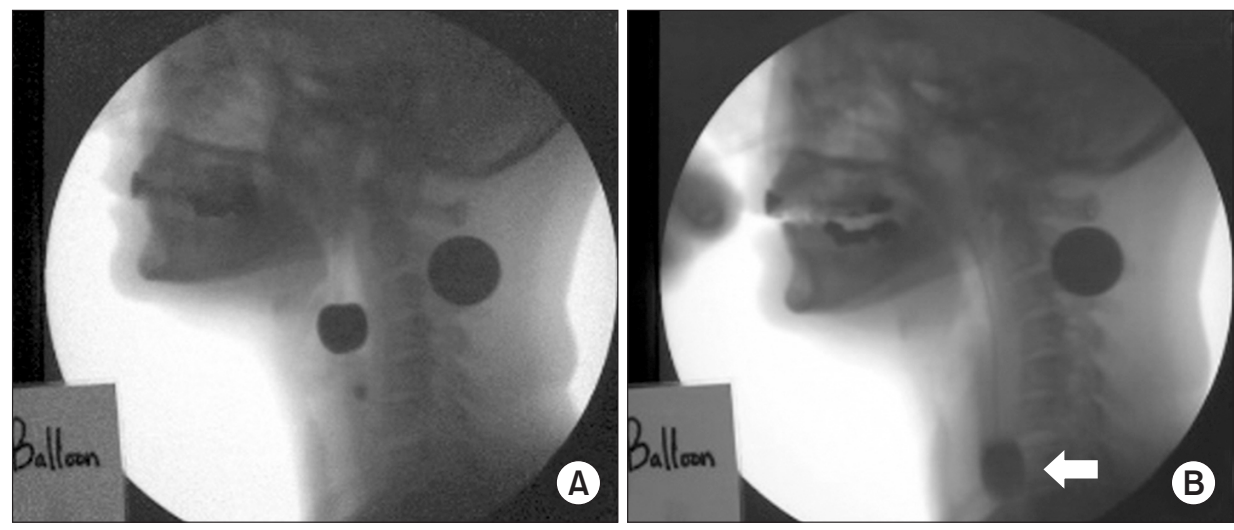

Fig. 2. Passage of the Foley balloon catheter in the videofluoroscopic swallowing study before and during pyriform sinus ballooning. A 12-Fr Foley catheter was placed in the pyriform sinus via a transnasal or transoral approach. It was filled with varying volumes of contrast media in increasing order: $0,1,2,3,4$ and $5 \mathrm{~mL}$, followed by meticulous monitoring of the swallowing process. Thus, we determined the greatest diameter of the balloon tolerated by the patients. (A) the passage of the balloon in the upper esophageal sphincter before and (B) during swallowing. The white arrow indicates the passage of the balloon.

catheter was inserted through the patients' nose or oral cavity and then placed in the pyriform sinus. It was filled with contrast media at varying volumes of $0,1,2,3,4$ and $5 \mathrm{~mL}$ in increasing order, which was followed by meticulous monitoring of the swallowing process (Fig. 2). Thus, we determined the greatest diameter of the balloon, which the patients tolerated. Furthermore, the location of the nostril before and after swallowing was marked on the Foley balloon catheter.

\section{Conventional rehabilitation protocol}

Conventional rehabilitation program for swallowing 
was performed by an occupational therapist. To minimize risks of developing aspiration as well as to reduce the pharyngeal remnant, we performed chin tuck, supraglottic swallow and Shaker exercise for the patients in the control group. In addition, we performed effortful or multiple swallowing and Mendelsohn maneuvers to improve bolus transport. Furthermore, we performed tongue strengthening exercise, tongue holding maneuver, laryngeal adduction and elevation exercises for oral motor facilitation [21].

The conventional rehabilitation was also combined with nutritional supplementation [22].

\section{Pyriform sinus ballooning protocol}

Non-fluoroscopy-guided pyriform sinus ballooning was performed by an occupational therapist under the supervision of a physician who was blinded to the current study. Pyriform sinus ballooning is based on voluntary swallowing or swallowing reflex, without deliberately relaxing the UES or inducing swallowing. The lubricant gel was applied to the tip of a 12-Fr Foley balloon catheter, which was inserted in the nasal cavity. In patients complaining of severe discomfort, it was done orally. Thus, the Foley balloon catheter was advanced to sites of marking immediately before swallowing on the VFSS. As the catheter reached the pyriform sinus, we performed inflation and deflation of air at a volume of approximately 0.5-1 mL until the repeated maneuver triggered the swallowing reflex. This approach was based on the volume of air equivalent to that of contrast media used to maximize the diameter of the balloon during the initial VFSS. We performed the pyriform sinus ballooning for 30 seconds maximum. Without completely ruling out the possibility of relaxation of the UES, we triggered the voluntary swallowing or swallowing reflex using the above maneuver.

Criteria for determining successful swallowing are as follows: the presence of downward force of the Foley balloon catheter according to the investigator's judgment; the exact match of the sites of marking before and after swallowing on the VFSS; and a lack of cough reflex. A transoral approach was also permitted for patients without gag reflex. In the non-cooperative patients with cognitive impairment, we induced the swallowing reflex through manual stimulation. We meticulously monitored the patients for complications such as epistaxis, gag reflex, tachycardia, bradycardia or syncope during the pro- cedure. Furthermore, balloon deflation was attempted to minimize the patients' discomfort.

\section{Rationale of sample size estimation}

According to Kim et al. [15], a comparison of post-treatment measurements between the two groups showed that the size of the mean pharyngeal remnant was $7.89 \pm 4.2 \mathrm{~mm}^{2}$. We hypothesized that a standard deviation of $4.2 \mathrm{~mm}^{2}$ resulted in clinical significance. Considering a significance level of $5 \%$, a statistical power of $90 \%$ and a drop-out rate of $30 \%$, we estimated a sample size of 21 per group.

\section{Statistical analysis}

Data were expressed as mean \pm standard deviation. Statistical analysis was carried out using the SPSS version 18.0 for Windows (SPSS Inc., Chicago, IL, USA). We compared the baseline characteristics between the two groups using the independent samples t-test. In addition, we also compared differences in changes in efficacy outcome measures at 4 weeks from baseline between the two groups using the Student t-test. A p-value of $<0.05$ was considered statistically significant.

\section{RESULTS}

\section{Baseline characteristics of the patients}

The 45 recruited patients were initially assigned to the trial group $(n=22)$ and the control group $(n=23)$. Among the patients in the trial group, 5 discontinued the study because of hospital-acquired pneumonia and 2 were lost to follow-up. In addition, 3 patients in the control group dropped out because of hospital-acquired pneumonia and 5 were lost to follow-up. Therefore, 15 patients were finally assigned to either the trial group or the control group $(\mathrm{n}=15)$.

Our clinical series of patients were divided equally into two groups: the trial group $(\mathrm{n}=15)$ and the control group $(n=15)$. They consisted of 12 men and 18 women, with a mean age of $58.5 \pm 16.5$ years. In addition, the patients' mRS score was $4.1 \pm 1.1$, NIHSS score $16.5 \pm 7.7$, time from the onset of stroke to the VFSS was $312.6 \pm 207.8$ days, and a K-MMSE score of 8.1 \pm 10.3 . Baseline characteristics of the patients are represented in Table 1. 
Table 1. Baseline characteristics of the patients

\begin{tabular}{|c|c|c|c|}
\hline & $\begin{array}{c}\text { Trial group } \\
(n=15)\end{array}$ & $\begin{array}{c}\text { Control } \\
\text { group }(n=15)\end{array}$ & $p$-value \\
\hline Age (yr) & $58.7 \pm 16.0$ & $58.4 \pm 16.9$ & 0.9605 \\
\hline Male-to-female ratio & $4: 11$ & $8: 7$ & 0.2597 \\
\hline mRS & $3.7 \pm 1.3$ & $4.5 \pm 0.6$ & 0.0391 \\
\hline NIHSS & $14.5 \pm 8.7$ & $18.6 \pm 5.8$ & 0.1401 \\
\hline $\begin{array}{l}\text { Time from the onset } \\
\text { of stroke to the } \\
\text { VFSS (day) }\end{array}$ & $354.1 \pm 248.7$ & $271 \pm 145.3$ & 0.2733 \\
\hline K-MMSE & $9.1 \pm 11.3$ & $7.1 \pm 9.0$ & 0.5961 \\
\hline \multicolumn{4}{|l|}{ Type of feeding } \\
\hline PEG & 5 & 2 & 0.2155 \\
\hline L-tube & 10 & 13 & 0.4283 \\
\hline \multicolumn{4}{|l|}{ Type of stroke } \\
\hline Ischemic & 8 & 3 & 0.2824 \\
\hline Hemorrhagic & 7 & 12 & 0.3610 \\
\hline \multicolumn{4}{|l|}{ Side of lesion } \\
\hline Right & 4 & 3 & 0.4206 \\
\hline Left & 6 & 4 & 0.3917 \\
\hline Multiple & 5 & 8 & 0.3766 \\
\hline \multicolumn{4}{|l|}{$\begin{array}{l}\text { Efficacy outcome } \\
\text { measures }\end{array}$} \\
\hline $\begin{array}{l}\text { Pharyngeal } \\
\text { remnant }\end{array}$ & $43.3 \pm 21.4$ & $44.2 \pm 13.2$ & 0.8907 \\
\hline $\begin{array}{l}\text { Pharyngeal transit } \\
\text { time }\end{array}$ & $9.2 \pm 4.0$ & $8.9 \pm 4.0$ & 0.8387 \\
\hline PAS & $4.8 \pm 1.8$ & $4.9 \pm 1.7$ & 0.8768 \\
\hline VDS & $69.5 \pm 8.9$ & $69.8 \pm 8.9$ & 0.9271 \\
\hline
\end{tabular}

Values are presented as mean \pm standard deviation. mRS, modified Rankin Scale; NIHSS, National Institutes of Health Stroke Scale; VFSS, videofluoroscopic swallowing study; K-MMSE, Korean version of modified MiniMental State Examination; PEG, percutaneous endoscopic gastrostomy; PAS, Penetration Aspiration Scale; VDS, Videofluoroscopic Dysphagia Scale.

\section{Efficacy outcomes}

As shown in Table 2, there were differences in outcome measures at 4 weeks from baseline between the two groups. There were significant differences in changes in the pharyngeal remnant, the pharyngeal transit time, the PAS scores, and the VDS scores at 4 weeks from baseline between the two groups $(\mathrm{p}<0.0001, \mathrm{p}=0.0092, \mathrm{p}<0.0001$, and $\mathrm{p}=0.0048$, respectively).

\section{Safety outcomes}

There were no TEAEs in our series.

\section{DISCUSSION}

Patients with stroke are at risk of paralysis of muscles due to degradation in motor skills and coordination ability. Paralysis of the cricopharyngeus muscle impairs the relaxation and contraction of the UES resulting in food remaining in the pharyngeal space without reaching the esophagus [22]. A sufficient degree of UES relaxation is an essential factor in the swallowing process. Strengthening of the suprahyoid muscle was effective in improving dysphagia arising from impaired UES opening [14]. Furthermore, physical exercise rehabilitation was also effective in improving the degree of UES relaxation via isotonic and isokinetic contraction of muscles involved [23,24].

Pyriform sinus ballooning was first attempted by Kim et al. [15] to improve the UES relaxation and the residue in the pyriform sinus in patients with PSD, by placing a 16-Fr Foley balloon catheter in the pharynx and adjusting the volume of food bolus during the swallowing process. Thus, this study eventually demonstrated that pyriform sinus ballooning is an effective modality although no control group was used. Similarly, our results also showed that there was a significant decrease in the

Table 2. Efficacy outcomes

\begin{tabular}{|c|c|c|c|c|c|c|c|}
\hline & \multicolumn{3}{|c|}{ Trial group $(n=15)$} & \multicolumn{3}{|c|}{ Control group $(n=15)$} & \multirow{2}{*}{ p-value } \\
\hline & Baseline & 4 weeks & $\Delta$ & Baseline & 4 weeks & $\Delta$ & \\
\hline Pharyngeal remnant & $43.3 \pm 21.4$ & $22.4 \pm 13.3$ & $20.9 \pm 8.1$ & $44.2 \pm 13.2$ & $33.8 \pm 11.6$ & $10.4 \pm 1.6$ & $<0.0001^{*}$ \\
\hline Pharyngeal transit time & $9.2 \pm 4.0$ & $4.9 \pm 2.0$ & $4.3 \pm 2.0$ & $8.9 \pm 4.0$ & $5.7 \pm 3.6$ & $3.2 \pm 0.4$ & $0.0001^{*}$ \\
\hline PAS & $4.8 \pm 1.8$ & $3.4 \pm 1.8$ & $1.4 \pm 0.0$ & $4.9 \pm 1.7$ & $4.0 \pm 1.5$ & $0.9 \pm 0.2$ & $<0.0001^{*}$ \\
\hline VDS & $69.5 \pm 8.9$ & $58.7 \pm 11.3$ & $10.8 \pm 2.4$ & $69.8 \pm 8.9$ & $60.9 \pm 8.8$ & $8.9 \pm 0.1$ & $0.0048^{*}$ \\
\hline
\end{tabular}

Values are presented as mean \pm standard deviation.

PAS, Penetration Aspiration Scale; VDS, Videofluoroscopic Dysphagia Scale.

${ }^{*} \mathrm{p}<0.05$ by Student t-test. 
pharyngeal remnant and pharyngeal transit time, using methods similar to those of Kim et al. [12,15]. However, we performed non-fluoroscopy-guided pyriform sinus ballooning and used a control group. Furthermore, our results are of significance in that a non-fluoroscopy-guided pyriform sinus ballooning is a sustainable intervention in patients with PSD, which contrasts with previous published studies in this series [12,15]. Furthermore, we marked the location of the balloon catheter before and after non-fluoroscopy-guided pyriform sinus ballooning to ensure that the accuracy of the blind approach was comparable to the fluoroscopy-guided procedure.

In the current study, we meticulously monitored for possible complications of balloon swallowing, to prevent TEAEs, which is also consistent with previous studies $[12,15]$.

Pharyngeal phase is initiated when the swallowing material passes the anterior facial arch through a voluntary mechanism based on lingual action. In addition, it is also initiated via an involuntary mechanism by the stimulation of the specific area of the pharynx and larynx. It normally occurs in healthy individuals for 1 second or shorter periods $[25,26]$. The tactile stimulation of the pyriform sinus with food or balloon is transmitted to the pharyngeal plexus innervating the pharyngeal mucosa. The pharyngeal plexus comprises the pharyngeal branch of the vagus nerve and pharyngeal branch of the glossopharyngeal nerve. The repetitive stimulation of the pyriform sinus through the inflation and deflation of the balloon may sensitize the pharyngeal plexus, and activate the nerve fibers innervating the pharyngo-laryngeal muscles. Our results showed a significant decrease in the pharyngeal transit time, which is consistent with previous studies [12,15].

Impaired swallow due to a variety of diseases induces voluntary compensation, including mechanical blending, the decreased size of the food bolus and tilting of head and neck. However, such compensation also occurs via an involuntary mechanism based on the adjustment of swallow apparatus. This pattern of pharyngeal swallow includes the posterior displacement of the tongue and larynx, the anterior displacement of the constrictor wall, the further upward and downward displacement of the larynx and the forward tilting of head. These phenomena occur as normal adaptation to the impaired swallow. In addition, a compensation failure leads to decompensa- tion resulting in ineffective swallowing and accumulation of residue in the pyriform sinus, penetration and aspiration.

Based on previous studies, we performed a 4-week course of exercise intervention for dysphagia rehabilitation using a Foley balloon catheter of specific size. Presumably, it leads to compensation based on involuntary mechanism and speculation that the pyriform sinus ballooning is an adaptation to normal swallowing from a therapeutic perspective. Furthermore, the decreased decompensation might improve swallowing ability and decrease the pharyngeal remnant [27]. In addition, we assumed that the pyriform sinus ballooning improves neuroplasticity based on a previous report suggesting that behavioral interventions were effective [28].

Pyriform sinus ballooning with the Foley balloon catheter allows adjustment of air volume and balloon size according to the swallowing ability of each patient, which plays a role in task-oriented training via repetitive swallowing. Kim et al. [12] reported a decrease in the pharyngeal remnant after swallowing as the size of balloon increased during intervention. Although it is impossible to directly measure the diameter in a clinical setting, it is possible to indirectly predict the swallowing function, which may therefore, play a role in setting treatment goals. Moreover, patients with PSD may be informed of changes in their swallowing functions at re-hospitalization and be motivated for the treatment. It may provide convenient treatment without specialized tools or diagnostic devices. Thus, it is a safe procedure without risks of aspiration pneumonia. It may provide a positive biofeedback. Habituation and sensitization based on a tactile, visual biofeedback during swallowing suggested that stimulation and intention-based input had a unique cortical swallowing mechanism [29]. In addition, another recent study also showed that electromyographic biofeedback was effective in restoring swallowing function and return to full oral food intake [30]. Based on these reports, it can be inferred not only that patients with PSD perceive changes in sensation during balloon swallowing every day or week but also carry elements associated with positive biofeedback. On the other hand, there were no significant differences in baseline K-MMSE scores between the two groups (trial group $9.1 \pm 11.3$ points; control group $7.1 \pm 9.0$ points). However, the results may differ depending on the severity of cognitive impairment, which 
requires further studies.

Limitations of the current study are as follows: we enrolled a relatively small number of patients. We could not therefore completely rule out the possibility of spontaneous recovery from PSD because of the relatively short period of study. We conducted the current study in the setting of a single, tertiary medical institution. We could not therefore completely rule out the possibility of selection bias. The observer was blinded to the ballooning procedure, but its outcomes may depend on his or her technical expertise. Even the small lesions may be a critical factor in patients with brainstem stroke because of clusters of reticular interneurons between cranial nerve nuclei, nerve tract and brainstem structure [31]. We failed to categorize patients based on the presence of brain lesions. There is a possibility that the effects of ballooning may differ in patients with brainstem stroke, which warrants further studies. No additional quantitative analysis of the pharyngeal peristalsis or UES relaxation via manometry or electrophysiological study of the pharyngo-laryngeal musculature was performed.

To summarize, our results revealed significant differences in changes associated with pharyngeal remnant, pharyngeal transit time, PAS scores and VDS scores at 4 weeks from baseline between the two groups $(\mathrm{p}<0.0001$, $\mathrm{p}=0.0001, \mathrm{p}<0.0001$ and $\mathrm{p}=0.0048$, respectively). There were no TEAEs in our series.

In conclusion, our results indicate that our 4-week rehabilitation program with pyriform sinus ballooning is an effective, safe and well-tolerated modality in patients with PSD. Pyriform sinus ballooning can be done by directly stretching and strengthening cricopharyngeus muscle. It is associated with fewer complications and induces swallowing reflex non-invasively and cost-effectively. However, further large-scale, long-term, multicenter studies are warranted to establish our results.

\section{CONFLICT OF INTEREST}

No potential conflict of interest relevant to this article was reported.

\section{REFERENCES}

1. Shi A, Tao Z, Wei P, Zhao J. Epidemiological aspects of heart diseases. Exp Ther Med 2016;12:1645-50.
2. Arnold M, Liesirova K, Broeg-Morvay A, Meisterernst J, Schlager M, Mono ML, et al. Dysphagia in acute stroke: incidence, burden and impact on clinical outcome. PLoS One 2016;11:e148424.

3. Gonzalez-Fernandez M, Ottenstein L, Atanelov L, Christian AB. Dysphagia after stroke: an overview. Curr Phys Med Rehabil Rep 2013;1:187-96.

4. Paik NJ, Kim IS, Kim JH, Oh BM, Han TR. Clinical validity of the functional dysphagia scale based on videofluoroscopic swallowing study. J Korean Acad Rehabil Med 2005;29:43-9.

5. Marik PE, Kaplan D. Aspiration pneumonia and dysphagia in the elderly. Chest 2003;124:328-36.

6. Sharma JC, Fletcher S, Vassallo M, Ross I. What influences outcome of stroke: pyrexia or dysphagia? Int J Clin Pract 2001;55:17-20.

7. Martino R, Pron G, Diamant N. Screening for oropharyngeal dysphagia in stroke: insufficient evidence for guidelines. Dysphagia 2000;15:19-30.

8. Speyer R, Baijens L, Heijnen M, Zwijnenberg I. Effects of therapy in oropharyngeal dysphagia by speech and language therapists: a systematic review. Dysphagia 2010;25:40-65.

9. Foley N, Teasell R, Salter K, Kruger E, Martino R. Dysphagia treatment post stroke: a systematic review of randomised controlled trials. Age Ageing 2008;37:25864.

10. Terré R, Mearin F. Effectiveness of chin-down posture to prevent tracheal aspiration in dysphagia secondary to acquired brain injury: a videofluoroscopy study. Neurogastroenterol Motil 2012;24:414-9.

11. Kang JH, Park RY, Lee SJ, Kim JY, Yoon SR, Jung KI. The effect of bedside exercise program on stroke patients with Dysphagia. Ann Rehabil Med 2012;36:51220.

12. Kim YK, Choi SS, Choi JH, Yoon JG. Effectiveness of rehabilitative balloon swallowing treatment on upper esophageal sphincter relaxation and pharyngeal motility for neurogenic dysphagia. Ann Rehabil Med 2015;39:524-34.

13. Zheng L, Li Y, Liu Y. The individualized rehabilitation interventions for dysphagia: a multidisciplinary case control study of acute stroke patients. Int J Clin Exp Med 2014;7:3789-94.

14. Shaker R, Easterling C, Kern M, Nitschke T, Massey B, Daniels S, et al. Rehabilitation of swallowing by exer- 
cise in tube-fed patients with pharyngeal dysphagia secondary to abnormal UES opening. Gastroenterology 2002;122:1314-21.

15. Kim JC, Kim JS, Jung JH, Kim YK. The effect of balloon dilatation through video-fluoroscopic swallowing study (VFSS) in stroke patients with cricopharyngeal dysfunction. J Korean Acad Rehabil Med 2011;35:23-6.

16. Kim YK, Lee SH, Lee JW. Effect of vallecular ballooning in stroke patients with dysphagia. Ann Rehabil Med 2017;41:231-8.

17. Kawada K, Kawano T, Sugimoto T, Matsui T, Okuda M, Ogo $\mathrm{T}$, et al. Observation of the pharynx to the cervical esophagus using transnasal endoscopy with blue laser imaging. In: Amornyotin S, editor. Endoscopyinnovative uses and emerging technologies. London: InTech; 2015.

18. Rensink M, Schuurmans M, Lindeman E, Hafsteinsdottir T. Task-oriented training in rehabilitation after stroke: systematic review. J Adv Nurs 2009;65:737-54.

19. Martin-Harris B, Brodsky MB, Michel Y, Ford CL, Walters B, Heffner J. Breathing and swallowing dynamics across the adult lifespan. Arch Otolaryngol Head Neck Surg 2005;131:762-70.

20. Kim J, Oh BM, Kim JY, Lee GJ, Lee SA, Han TR. Validation of the videofluoroscopic dysphagia scale in various etiologies. Dysphagia 2014;29:438-43.

21. Vose A, Nonnenmacher J, Singer ML, Gonzalez-Fernandez M. Dysphagia management in acute and subacute stroke. Curr Phys Med Rehabil Rep 2014;2:197206.

22. Alfonsi E, Merlo IM, Ponzio M, Montomoli C, Tassorelli C, Biancardi C, et al. An electrophysiological approach to the diagnosis of neurogenic dysphagia: implications for botulinum toxin treatment. J Neurol Neurosurg Psychiatry 2010;81:54-60.

23. Ferdjallah M, Wertsch JJ, Shaker R. Spectral analysis of surface electromyography (EMG) of upper esophageal sphincter-opening muscles during head lift exercise. J Rehabil Res Dev 2000;37:335-40.

24. Rommel N, Hamdy S. Oropharyngeal dysphagia: manifestations and diagnosis. Nat Rev Gastroenterol Hepatol 2016;13:49-59.

25. de Lama Lazzara G, Lazarus C, Logemann JA. Impact of thermal stimulation on the triggering of the swallowing reflex. Dysphagia 1986;1:73-7.

26. Matsuo K, Palmer JB. Anatomy and physiology of feeding and swallowing: normal and abnormal. Phys Med Rehabil Clin N Am 2008;19:691-707.

27. Buchholz DW, Bosma JF, Donner MW. Adaptation, compensation, and decompensation of the pharyngeal swallow. Gastrointest Radiol 1985;10:235-9.

28. Martin RE. Neuroplasticity and swallowing. Dysphagia 2009;24:218-29.

29. Humbert IA, Joel S. Tactile, gustatory, and visual biofeedback stimuli modulate neural substrates of deglutition. Neuroimage 2012;59:1485-90.

30. Crary MA, Groher ME. Basic concepts of surface electromyographic biofeedback in the treatment of dysphagia: a tutorial. Am J Speech Lang Pathol 2000;9:116-25.

31. Huckabee ML, Cannito MP. Outcomes of swallowing rehabilitation in chronic brainstem dysphagia: a retrospective evaluation. Dysphagia 1999;14:93-109. 